\title{
miR-219-5p plays a tumor suppressive role in colon cancer by targeting oncogene Sall4
}

\author{
JI CHENG, RUI DENG, PENG ZHANG, CHUANQING WU, KE WU, LIANG SHI, \\ XINGHUA LIU, JIE BAI, MEIZHOU DENG, XIAOMING SHUAI, \\ JINBO GAO, GUOBIN WANG and KAIXIONG TAO \\ Department of Gastrointestinal Surgery, Union Hospital, Tongji Medical College, \\ Huazhong University of Science and Technology, Wuhan, Hubei, P.R. China
}

Received June 10, 2015; Accepted July 15, 2015

DOI: $10.3892 / o r .2015 .4168$

\begin{abstract}
Sall4 is a novel oncogene found upregulated in several malignancies including colon cancer. However, its upstream regulatory miRNA is still undefined. miR-219-5p is regarded as a tumor-related miRNA in cancer research. Nevertheless, its actual role of whether inhibiting or promoting tumorigenesis is unclear in colon cancer. Potential interaction between Sall4 and miR-219-5p is predicted by TargetScan. CCK-8 test was used for evaluation of cell proliferation and cell survival rates. Western blot analysis and real-time PCR were applied for detection of target molecules. Luciferase assay was a direct confirmation of mutual interaction. Wound healing assay and Transwell assay were conducted for cell migration and invasion tests. Flow cytometry was used for cell apoptosis analysis. Tissue specimens and cell lines were explored for miR-219-5p inhibition on colon cancer proliferation, migration, invasion, apoptosis and drug resistance by targeting Sall4. The results show that miR-219-5p inhibited carcinogenesis of colon cancer by targeting oncogene Sall4.
\end{abstract}

\section{Introduction}

Colon cancer is one of the most lethal malignancies worldwide, with its incidence rate rising each year due to the lifestyle innovation and environment modification, especially in the rapid urbanization regions such as China. Modern multimodality treatments, including primary surgery resection and other adjuvant therapies, have synergistically improved the overall 5-year survival rate to $\sim 60 \%(1,2)$. However, distant metastasis and drug resistance restrain the efficacy of current

Correspondence to: Professor Kaixiong Tao, Department of Gastrointestinal Surgery, Union Hospital, Tongji Medical College, Huazhong University of Science and Technology, 1277 Jiefang Avenue, Wuhan, Hubei, P.R. China

E-mail: kaixiongtaowhuh@126.com

Key words: Sall4, miR-219-5p, colon cancer, miRNA medications. These obstacles make it essential for the investigation of mechanisms that contribute to the poor prognosis of advanced and resistant cases.

MicroRNA (miRNA) is constituted by an endogenous conserved class of small, non-coding RNAs exerting regulatory function on multiple gene expressions via translational suppression and mRNA degradation. Correlations between miRNAs and carcinogenesis of colon cancer have been verified by emerging evidence (3). By means of blocking target oncogenes or tumor suppressor genes, miRNAs act contradictory roles in modulating cancer proliferation, metastasis, apoptosis and drug resistance (4).

miR-219-5p has been reported to have a role in many biological procedures including myocardial regeneration (5) and lymphoblast immortalization (6). Compelling evidence suggests that miR-219-5p serves as a tumor manipulator in various types of cancers, attenuating malignant features of liver cancer (7), papillary thyroid carcinoma (8) and glioblastoma (9) by targeting downstream oncogenic molecules. Nevertheless, whether miR-219-5p is aberrantly expressed and associated with oncogenesis in colon cancer remains unclear.

Sall4 is a novel oncogene mediating origination and development in different types of cancers. Its abnormal overexpression in colon cancer leads to tumor progression and metastasis (10), and Sall4 has become a new biomarker for early diagnosis among colon malignancies (11). Several upstream pathways have been identified as modulators of Sall4, such as Wnt $/ \beta$-catenin signaling (12), and certain miRNAs, including miR-107 (13), directly regulate Sall4 expression, controlling cell proliferation and invasiveness. In spite of all above evidence, the regulatory mechanisms against Sall4 in colon cancer remain ill-defined.

In the present study, we investigated the aberrant expression of miR-219-5p and Sall4 in colon cancer specimens, and confirmed that Sall4 was the direct target of miR-219-5p. Additionally, by aid of gain and loss of function assays, miR-219-5p was observed to play an inhibitory effect on cell proliferation, invasion and drug resistance. Our findings demonstrate that miR-219-5p has potential clinical value against colon cancer and may be an important therapy target in future treatments. 


\section{Materials and methods}

Tissue specimens. All human related studies were approved by the Ethics Committee of Union Hospital, Tongji Medical College, Huazhong University of Science and Technology and were performed in accordance with the ethical standards of Declaration of Helsinki. Prior to the collection of specimens, a written informed consent was given by each person involved.

The tissue specimens were collected from 20 patients who had undergone surgical resection in the Gastroenterology Department, Union Hospital, Tongji Medical College, Huazhong University of Science and Technology in 2014. All patients had surgical procedures prior to chemotherapy or radiation therapy. Post-surgical pathology had verified the resection tissues as colon cancer tissues and adjacent normal tissues from each patient were applied as controls.

Cell culture. Normal human colon epithelial cell line NCM460 was kindly donated by our laboratory instructor, and colon cancer cell line HT-29, Caco-2, DLD-1, SW480, SW620 were purchased from the American Type Culture Collection (ATCC; Manassas, VA, USA). Dulbecco's modified Eagle's medium (DMEM; Life Technologies, Grand Island, NY, USA) supplemented with $10 \%$ fetal bovine serum (FBS; SJQ, Zhejiang Tianhang Biotechnology Ltd., Hangzhou, China) was applied for normal culture and the cells were incubated in a humidified incubator at $37^{\circ} \mathrm{C}$ with $5 \% \mathrm{CO}_{2}$.

Quantitative real-time PCR. Total RNA was isolated from tissue specimens and cultured cells using TRIzol reagent (Takara, Dalian, China). miR-219-5p was polyadenylated by One-Step PrimeScript miRNA cDNA synthesis kit (D350A; Takara) under the manufacturer's protocol. Then cDNA was quantified using SYBR Premix EX Taq II (DRR081; Takara) for real-time PCR quantification in Applied Biosystems 7500 Real-Time PCR Systems (Applied Biosystems, Foster City, CA, USA) under standard procedures. U6 was used as an internal reference for miR-219-5p. The forward primer sequence of miR-219-5p was: 5'-CGGTGATTGTCCAAACG CAATTC-3'; the reverse primer was Uni-miR qPCR Primer provided by One-Step PrimeScript miRNA cDNA synthesis kit (D350A; Takara). All reactions were perfomed in triplicate. The $2^{-\Delta \Delta C T}$ method was applied for relative expression quantification.

Western blotting. Total proteins were extracted from tissue specimens and cultured cells using RIPA buffer. BCA assay was applied for protein concentration evaluation using Pierce ${ }^{\mathrm{TM}}$ BCA protein assay kit (23225; Life Technologies) following the manufacturer's protocol. Total protein $(40 \mu \mathrm{g} / \mathrm{lane})$ was separated on $10 \%$ SDS polyacrylamide gels and transferred onto PVDF membranes. After blocking in 5\% fat-free milk for $2 \mathrm{~h}$, the membranes were then incubated with primary antibody overnight at $4^{\circ} \mathrm{C}$. The primary antibodies used were: anti-Sall4 (ab29112; Abcam, Cambridge, MA, USA); anti-GAPDH (ab37168; Abcam); anti-p21 (sc-397; Santa Cruz Biotechnology, Santa Cruz, CA, USA); anti-cyclin D1 (sc-753; Santa Cruz Biotechnology); anti-MMP-9 (sc-10737; Santa Cruz Biotechnology); anti-E-cadherin (sc-7870; Santa
Cruz Biotechnology); anti-N-cadherin (sc-7939; Santa Cruz Biotechnology); anti-BAX (sc-493; Santa Cruz Biotechnology); anti-cleaved caspase-3 (sc-22171-R; Santa Cruz Biotechnology); anti-cleaved caspase-9 (\#7237; Cell Signaling Technology, Danvers, MA, USA); anti-P-gp (ab129450; Abcam); antiMRP1 (sc-13960; Santa Cruz Biotechnology). After washing with TBST, the membrane was incubated with anti-rabbit IgG-HRP secondary antibody (074-1506; KPL, Gaithersburg, MD, USA) for $2 \mathrm{~h}$. Protein bands were visualized by X-ray film using Pierce ${ }^{\mathrm{TM}}$ ECL Western blotting substrate (32209; Life Technologies).

Dual-luciferase reporter assay. miR-219-5p mimic, inhibitor, negative control and wild-type or mutant pGL3-Sall4-3'UTR were co-transfected into cultured cells seeded in a 96-well. Dual-luciferase reporter assay system (Promega, Madison, WI, USA) was applied for analyzing luciferase activity $48 \mathrm{~h}$ post-transfection. The firefly and Renilla luciferase activities were measured and firefly luciferase activity was normalized to the Renilla luciferase activity. All experiments were conducted in triplicate.

Cell transfection. miR-219-5p mimic, miR-219-5p inhibitor, scrambled negative control and siRNA of Sall4 were synthesized by Guangzhou RiboBio Co., Ltd. (Guangzhou, China); pcDNA3.1-Sall4 expression vector was constructed by CIYA Technologies (Wuhan, China). Cells were incubated in 6-well plates at a density of $10^{5}$. When the cell confluence reached 70\%, miR-219-5p mimic, miR-219-5p inhibitor, scrambled negative control, siRNA of Sall4 and pcDNA3.1-Sall4 expression vector were transfected respectively or co-transfected to cells in different groups with Lipofectamine ${ }^{\circledR} 2000$ transfection reagent (11668-019; Life Technologies) following standard procedures. The testing of transfection efficiency on qRT-PCR and collection of cells for assays were conducted $48 \mathrm{~h}$ posttransfection.

Cell proliferation assay. Cells were seeded in 96-well plates in a concentration of $10^{4} /$ well, each group had 6 parallel wells. CCK-8 kit (Dojindo Laboratories, Kumamoto, Japan) was applied to detect the proliferation of cells after 24-, 48- and 72-h incubation. CCK-8 reagent (10 $\mu \mathrm{l})$ was added into each well at different time-points before 2 -h incubation. Then the absorbance at $450 \mathrm{~nm}$ was measured. Three independent experiments were conducted.

Cell migration and invasion assays

Wound healing assay. An artificial wound was made by a $200-\mu 1$ pipette tip scratching on the well surface. To eliminate proliferation effect, basic DMEM medium without fetal bovine serum was used to culture cells for $24 \mathrm{~h}$ until evaluating the final efficacy.

Transwell assay. Cell invasion was examined using 24-well Matrigel invasion chamber with pore size $8 \mu \mathrm{m}$ (Corning, Incorporated, Corning, NY, USA). Cells $\left(10^{5}\right)$ were added to the upper compartment of the chamber with serum-free medium, while the lower compartment was filled with complete medium with $10 \%$ fetal bovine serum as a chemoattractant. After 48-h incubation, non-invading cells on the 


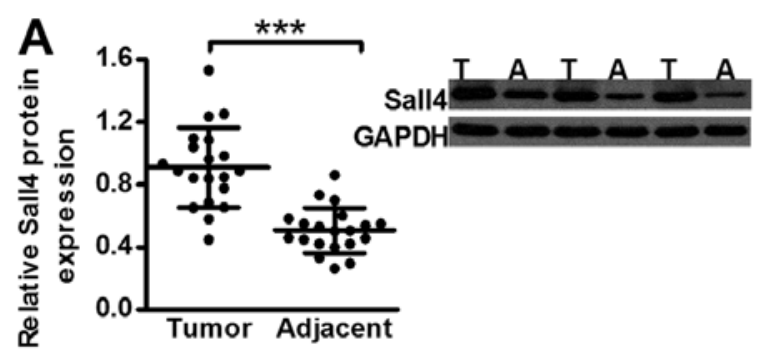

D
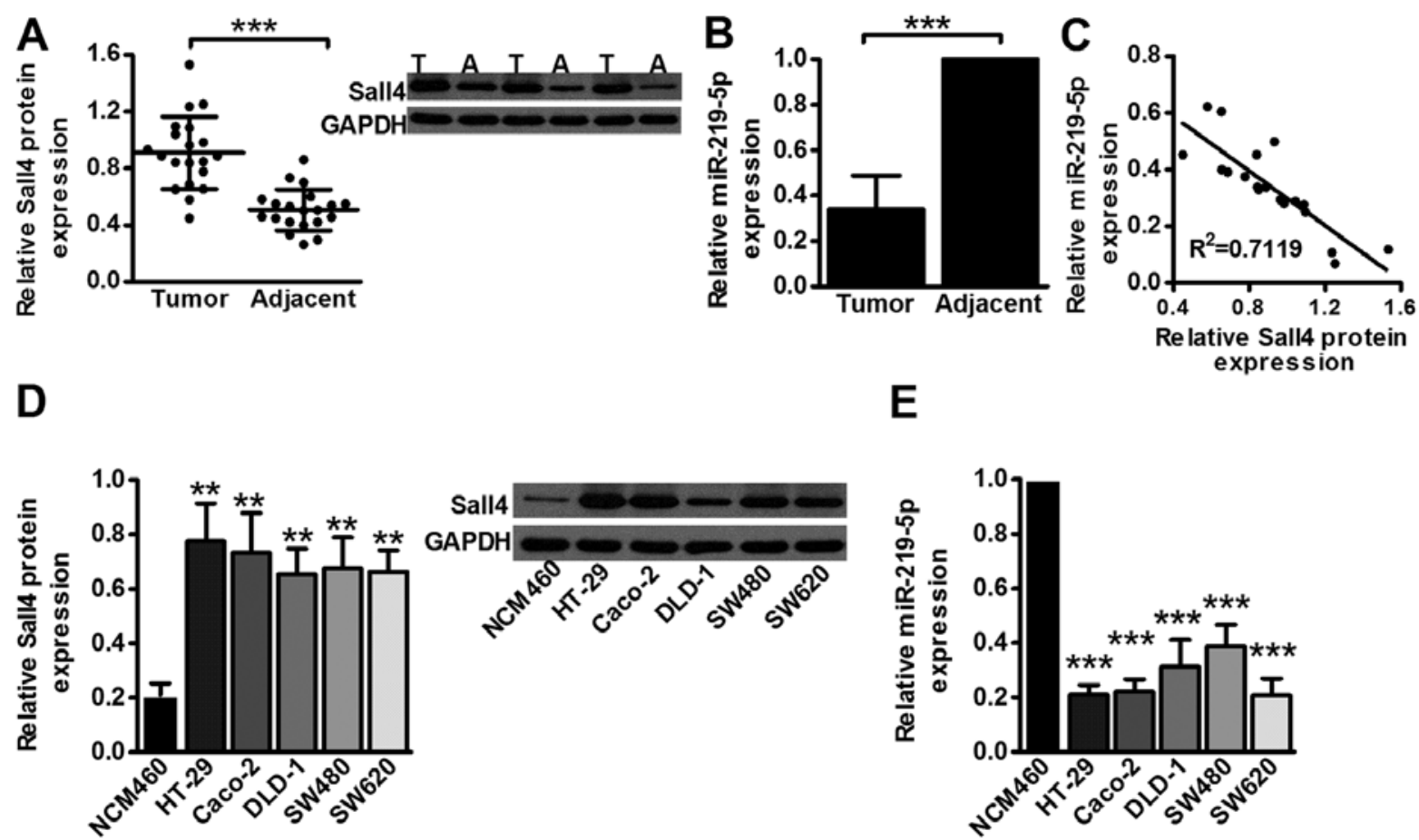

E

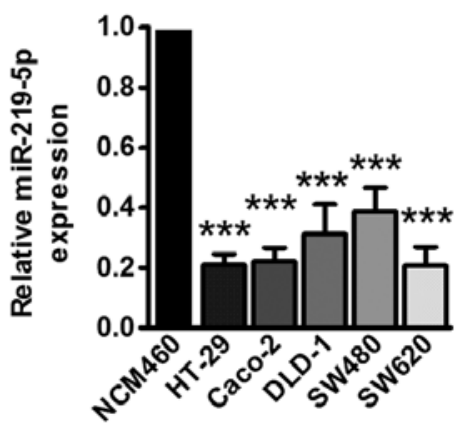

Figure 1. The expression of Sall4 and miR-219-5p in colon cancer specimens and cell lines (A) Left panel, relative Sall4 expression in tumor and adjacent normal tissues of 20 patients. Right panel, three representative western blot figures among all specimens on Sall4 expression (T, tumor; A, adjacent); (B) relative miR-219-5p expression in tumor and adjacent normal tissues of 20 patients; (C) linear correlation analysis of Sall4 and miR-219-5p expression on tissue specimens; (D) left panel, relative Sall4 expression of colon cancer cell lines compared to normal colon epithelial cell line NCM460. Right panel, representative western blot figure; (E) relative miR-219-5p expression of colon cancer cell lines compared to normal colon epithelial cell line NCM460. Column, means; Bar, $\mathrm{SD} ;{ }^{*} \mathrm{P}<0.05 ;{ }^{* *} \mathrm{P}<0.01 ;{ }^{* * *} \mathrm{P}<0.001$.

upper surface were wiped with a cotton swab. Ethanol $(95 \%)$ and $0.1 \%$ crystal violet solution were used to fix and stain the invasive cells. The numbers of invasive cells were counted from four randomly selected fields (magnification, x200). All experiments were conducted in triplicate.

\section{Cell cycle and apoptosis analysis}

Cell cycle. The cells were collected and fixed in $70 \%$ ethanol overnight at $-20^{\circ} \mathrm{C}$, then treated with DNA staining solution containing $3.4 \mathrm{mM}$ Tris- $\mathrm{Cl}(\mathrm{pH} 7.4)$, propodium iodide, $0.1 \%$ Triton X-100 buffer and $100 \mathrm{mg} / \mathrm{ml}$ RNase A. Cell cycle analysis was conducted in FACS flow cytometry (BD Biocsiences, San Jose, USA).

Apoptosis. Annexin V-FITC/PI dual staining kit (KGA108; Nanjing KeyGen Biotech Co., Ltd., Nanjing, China) was used for evaluation of apoptosis. Cells $\left(3 \times 10^{5}\right)$ were collected before analyzing by FACS flow cytometry (BD Biocsiences) following recommended steps. All experiments were performed in triplicate.

Drug resistance analysis. CCK- 8 assay was used for analyzing the cell viability after exposure to each drug for 72 h. Fluorouracil (Yabao Pharmaceutical Co., Ltd., Beijing, China) and oxaliplatin (Hangzhou Sanofi-Aventis Minsheng Co., Ltd., China) were used to confirm the drug sensitivity of the different cells.

Statistical analysis. The linear analysis between miR-219-5p and Sall4 in specimens was explored by Spearman's correla- tion. Other data were analyzed by the Student's t-test. $\mathrm{P}<0.05$ was regarded as statistically significant. Excel software and GraphPad Prism 5 were used for statistical analysis.

\section{Results}

Sall4 expression is elevated and miR-219-5p is downregulated in colon cancer tissue specimens and cell lines compared to controls. By analyzing specimens from 20 colon cancer patients, Sall4 expression in the tumor tissues was found elevated compared to adjacent normal tissues $(\mathrm{P}<0.001$; Fig. 1A), while miR-219-5p level was reduced in the malignant tissues compared to controls $(\mathrm{P}<0.001$; Fig. 1B). Then, the linear regression analysis displayed a possible relevance between Sall4 and miR-219-5p in tissue specimens with R2=0.7119 (Fig. 1C). Cell line assays coordinated with tissue analysis indicating that Sall4 level was elevated in colon cancer cell line HT-29 ( $\mathrm{P}<0.01)$, Caco-2 $(\mathrm{P}<0.01)$, DLD-1 $(\mathrm{P}<0.01)$, SW480 $(\mathrm{P}<0.01)$ and SW620 $(\mathrm{P}<0.01)$ compared to normal colon epithelial cell line NCM460 (Fig. 1D) while miR-219-5p level was decreased in HT-29 ( $\mathrm{P}<0.001)$, Caco-2 $(\mathrm{P}<0.001)$, DLD-1 $(\mathrm{P}<0.001)$, SW480 $(\mathrm{P}<0.001)$ and SW620 $(\mathrm{P}<0.001)$ (Fig. 1E). The results revealed that Sall4 expression was elevated in cancer tissues while miR-219-5p was reversely downregulated. There was potential interaction between Sall4 and miR-219-5p based on expression analysis.

Sall4 is a direct target of miR-219-5p. miR-219-5p was predicted by TargetScan as a potential regulator of oncogene Sall4 (Fig. 2A). To clarify the interplay between Sall4 and 
A

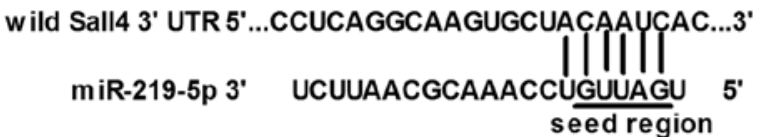

mutant Sall4 3' UTR 5'...CCUCAGGCAAGUGCUACACGAAC....3'
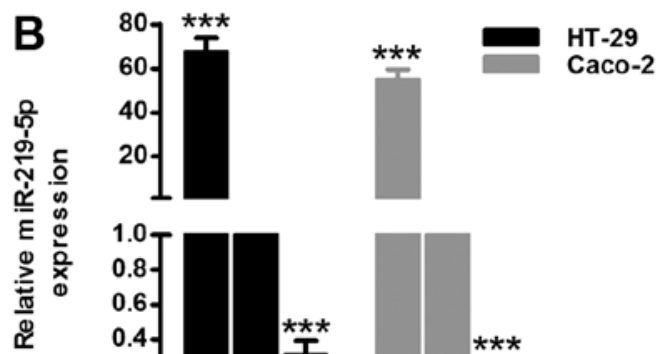

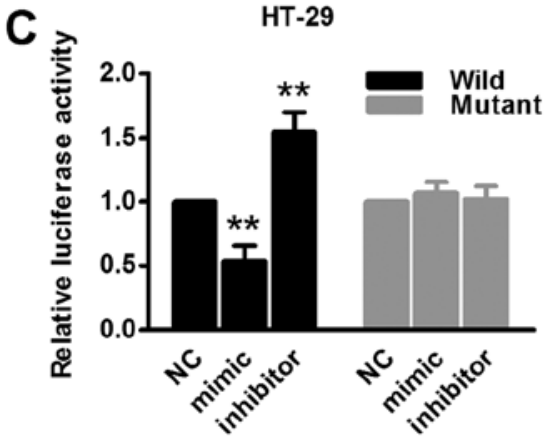

E

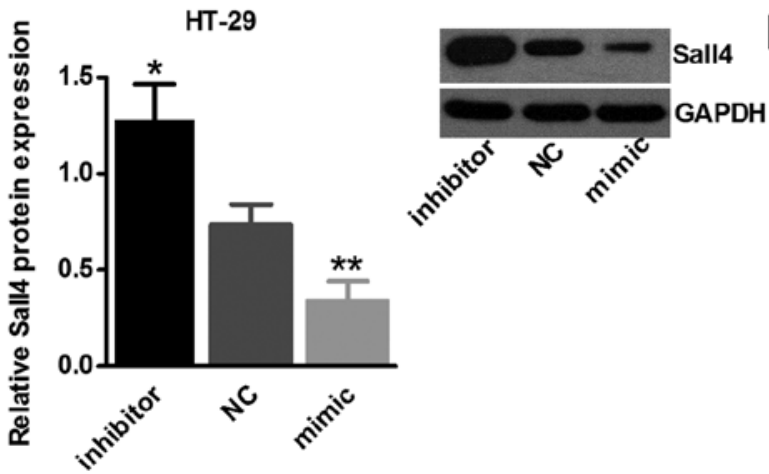

D

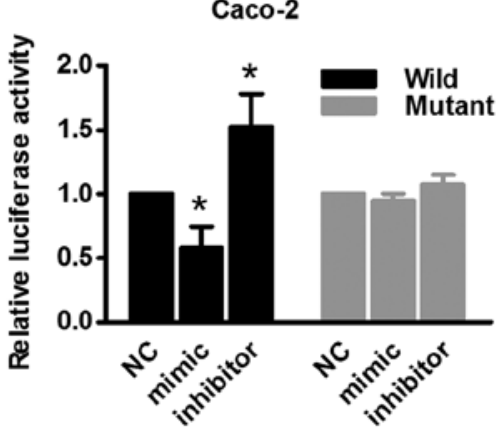

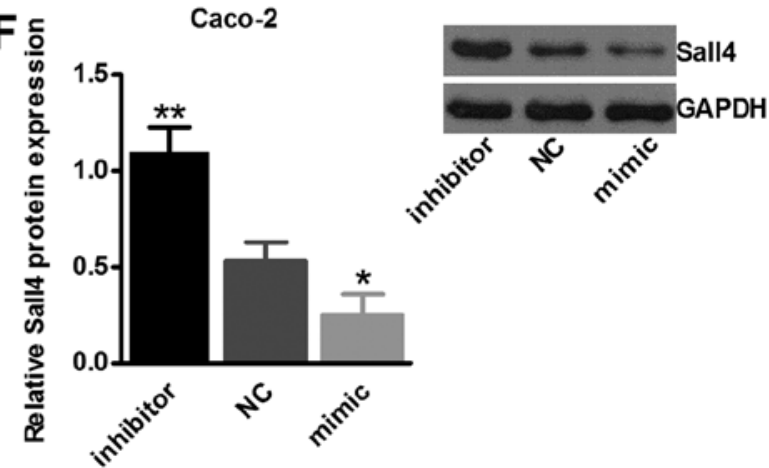

Figure 2. Sall4 was a direct target of miR-219-5p. (A) Sequences of Sall4 3'UTR and miR-219-5p according to TargetScan prediction; (B) transfection efficiency detection in two cell lines; (C and D) relative luciferase activity on wild or mutant type of Sall4 3'UTR of mimic, inhibition and negative control groups in the cell lines; (E and F) relative Sall4 expression level of mimic, inhibition and negative control groups in the cell lines. Column, means; Bar, SD. ${ }^{*} \mathrm{P}<0.05 ;{ }^{* *} \mathrm{P}<0.01 ;{ }^{* * *} \mathrm{P}<0.001$.

miR-219-5p, we used the dual luciferase assay in HT-29 and Caco-2 cell lines. Transfection efficiency detection is shown in Fig. 2B, miR-219-5p mimic upregulated its expression dramatically $(\mathrm{P}<0.001)$ and vice versa $(\mathrm{P}<0.001)$. Dual luciferase assay revealed that upregulation of miR-219-5p inhibited luciferase activity compared to negative control group in wild-type 3'UTR, but not in mutant type, which strongly proved that miR-219-5p could indeed interact with Sall4 3'UTR sequence (Fig. 2C and D; P<0.05). Moreover, western blot assay additionally supported the results of dual luciferase assay that upregulation of miR-219-5p inhibited Sall4 expression and vice versa (Fig. 2E and F; P $<0.05$ ), confirming Sall4 was a direct target of miR-219-5p.

miR-219-5p inhibited colon cancer proliferation and G0/G1 cell cycle arrest by targeting Sall4. To accurately find out the interaction of miR-219-5p and Sall4 on colon cancer proliferation, we divided HT-29 and Caco-2 into five groups respectively: 1, miR-219-5p mimic and Sall4 activation vector co-transfection; 2, miR-219-5p; 3, negative control; 4, miR-219-5p inhibitor; 5, miR-219-5p inhibitor and siRNA of Sall4 co-transfection. By CCK-8 test, we discovered that in both cell lines, miR-219-5p inhibited cell proliferation dramatically compared to negative control, which then could be antagonized by Sall4 activation $(\mathrm{P}<0.05$; Fig. $3 \mathrm{~A}$ and $\mathrm{B})$. Additionally, reduction on miR-219-5p expression increased cell proliferation and those effects were inhibited by Sall4 siRNA transfection $(\mathrm{P}<0.05$; Fig. $3 \mathrm{~A}$ and $\mathrm{B})$. In cell cycle analysis, both cell lines displayed G0/G1 arrest and S phase percentage reduction by miR-219-5p activation and vice versa $(\mathrm{P}<0.05$; Fig. $3 \mathrm{C}$ and $\mathrm{D}) . \mathrm{p} 21$ was a G1 phase checkpoint protein acting as a tumor suppressor while cyclin D1 promoted cells into $\mathrm{S}$ phase functioning as a proliferative role. Moreover, by activation of miR-219-5p, p21 increased its expression while cyclin D1 decreased, these results could be antagonized by targeting Sall4 ( $\mathrm{P}<0.05$; Fig. $3 \mathrm{E}$ and F). All the outcomes confirmed that miR-219-5p inhibited colon cancer proliferation and G0/G1 cell cycle arrest by targeting Sall4. 
A

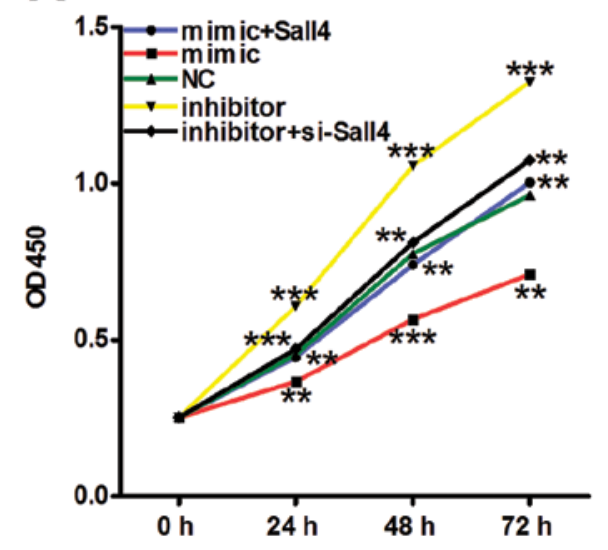

B

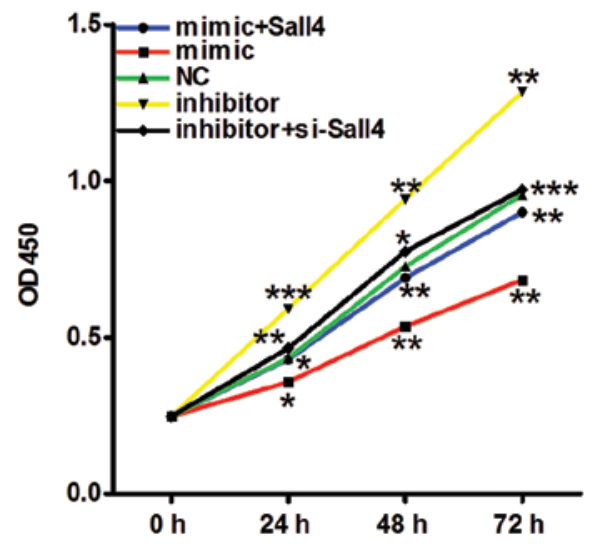

HT-29
C

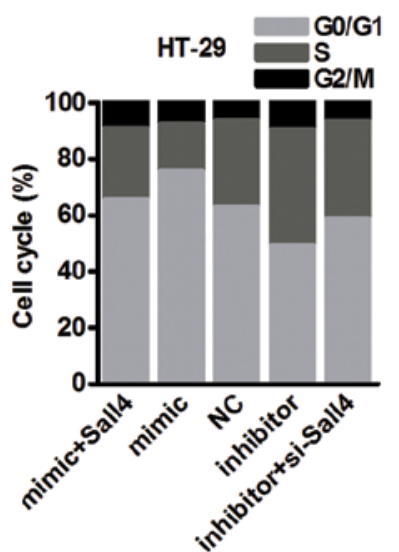

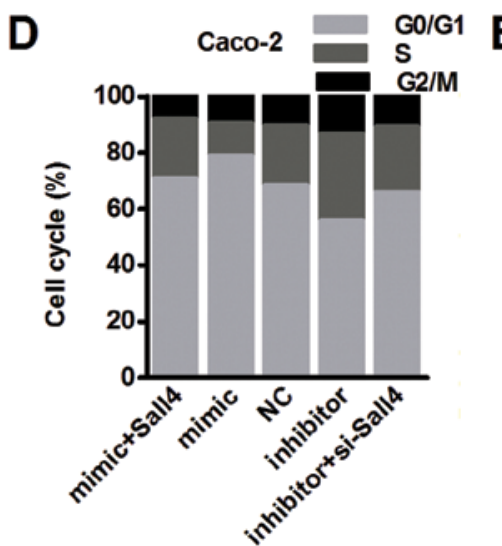
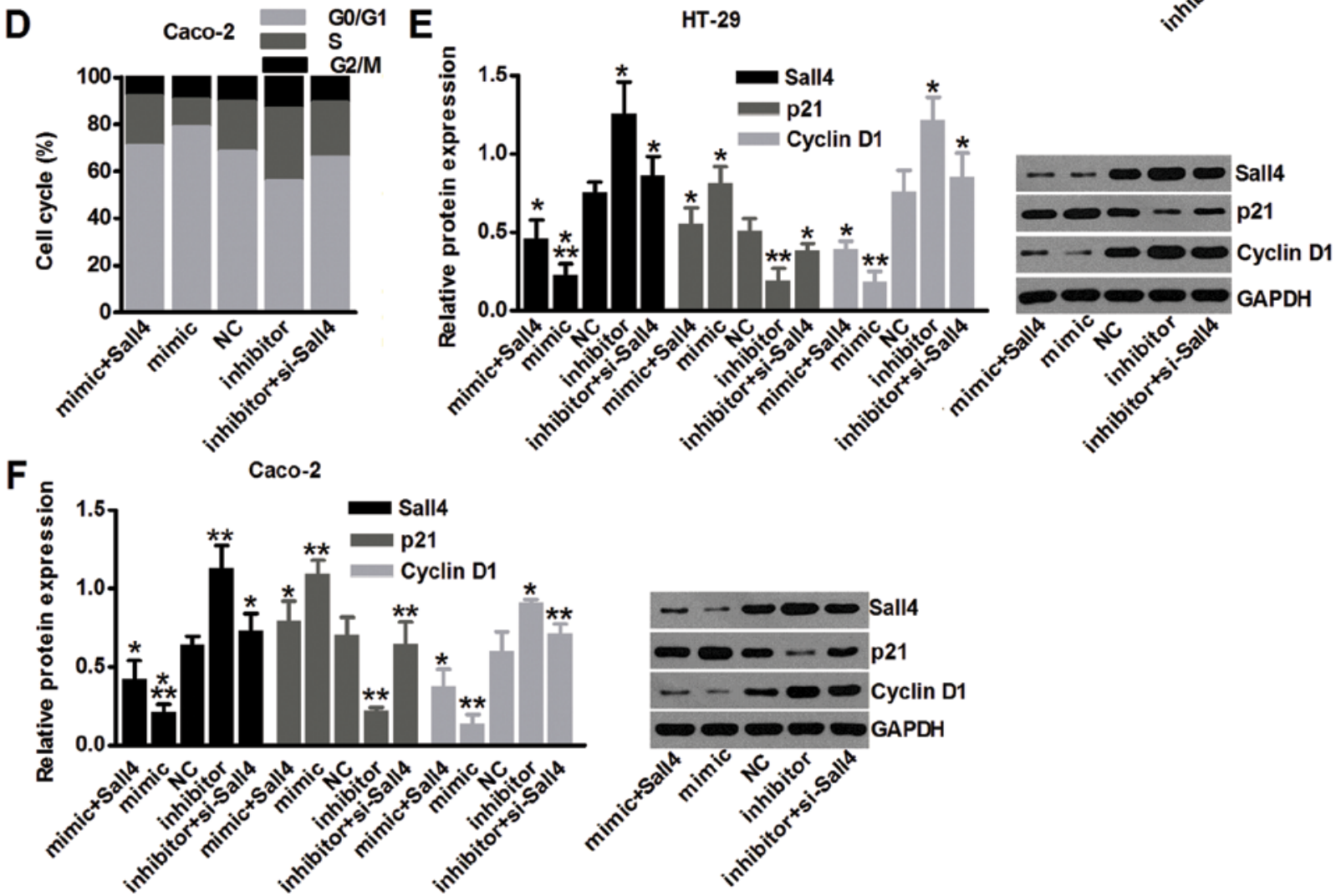

Figure 3. miR-219-5p inhibits colon cancer proliferation and G0/G1 cell cycle arrest by targeting Sall4. (A and B) Cell proliferation detection after 24,48 and $72 \mathrm{~h}$ among five different groups in both cell lines by CCK-8 test; (C and D) percentage of cell cycle analysis among five different groups in both cell lines; (E and F) cell cycle-related protein expression among five different groups in both cell lines. Right portions are representative western blot figures; mutual comparison relationships: mimic+Sall4 vs. mimic; mimic vs. NC; NC vs. inhibitor; inhibitor vs. inhibitor+si-Sall4. Column, means; Bar, SD. "P<0.05; ** P<0.01; ${ }^{* * * *} \mathrm{P}<0.001$

miR-219-5p inhibits colon cancer migration and invasion by targeting Sall4. Wound healing assay was routinely used to analyze cell migration and Transwell assay was applied as a classical measure for cancer invasiveness. Our experiments confirmed that cell migration and invasion were reduced dramatically by miR-219-5p activation, which were promoted by miR-219-5p inhibition. The Sall4 expression level was able to influence the efficacy, suggesting miR-219-5p inhibited colon cancer migration and invasion by targeting Sall4 $(\mathrm{P}<0.05$; Fig.4C and F). MMP-9, E-cadherin and N-cadherin are representative invasion-related protein in cancer research increasing their expression in more invasive cases (E-cadherin decreasing). By western blot assay, the expression level of targeted protein revealed similar results to the above experiments $(\mathrm{P}<0.05$; Fig. $4 \mathrm{G}$ and $\mathrm{H})$.

miR-219-5p induces colon cancer apoptosis by targeting Sall4. Our experiments confirmed by flow cytometry analysis, that miR-219-5p strongly increased cell apoptosis rate, while the rate was inhibited by miR-219-5p inhibition $(\mathrm{P}<0.05$; Fig. $5 \mathrm{C}$ and D). Sall4 was identified as a target of miR-219-5p-induced apoptosis in colon cancer cell lines. BAX, cleaved caspase-3 

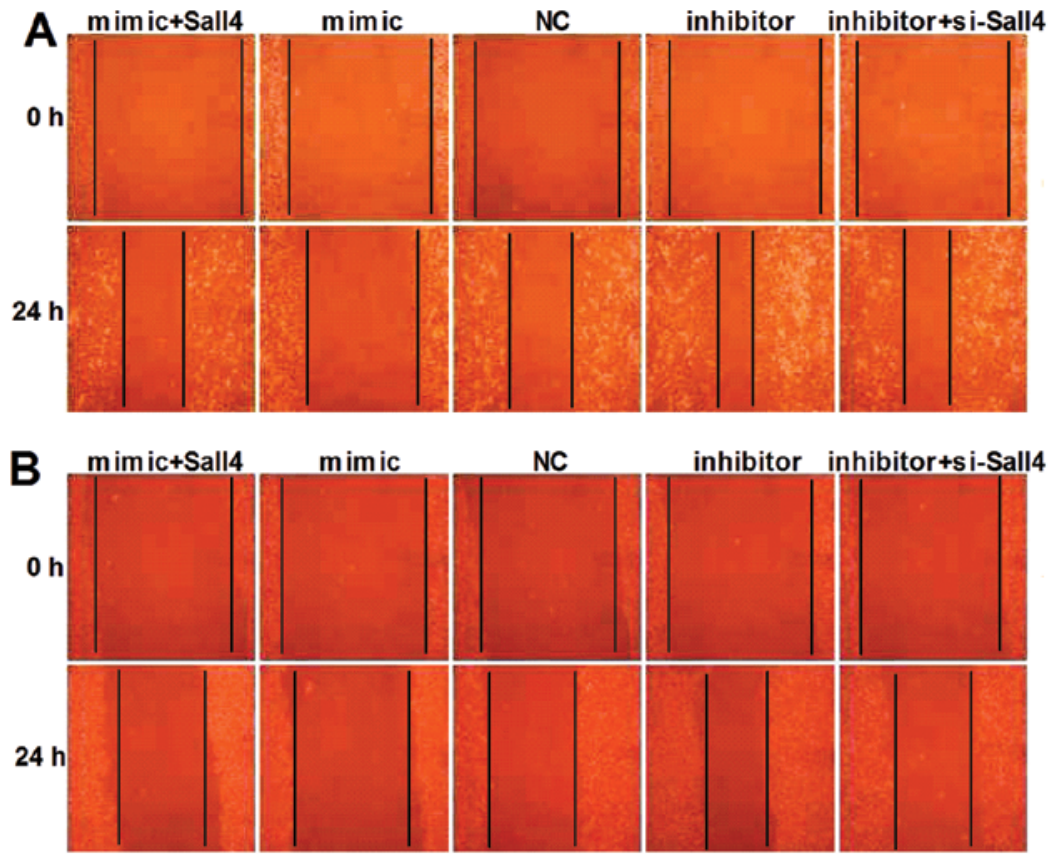

D
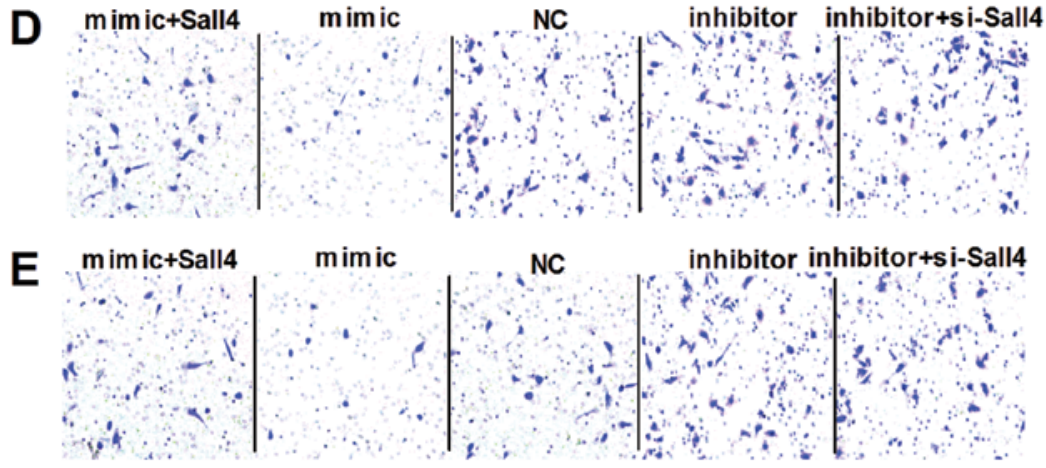

G

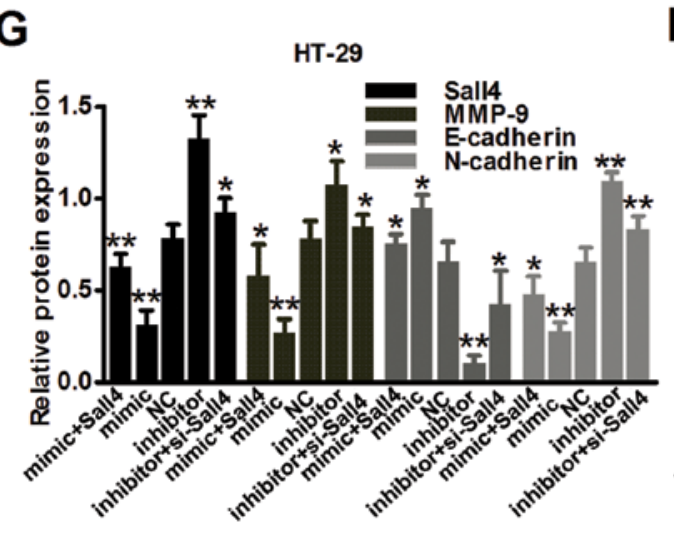

inhibitor inhibitor+si-Sall4
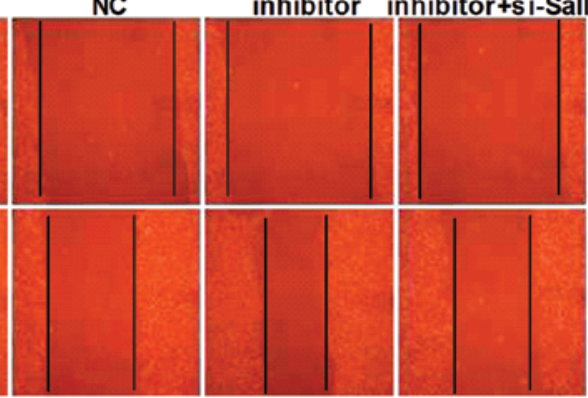

inhibitor inhibitor+si-Sall4

\section{mimic}
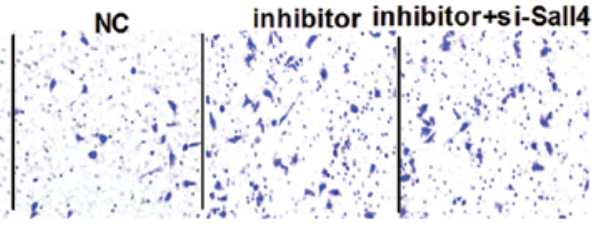

Caco-2
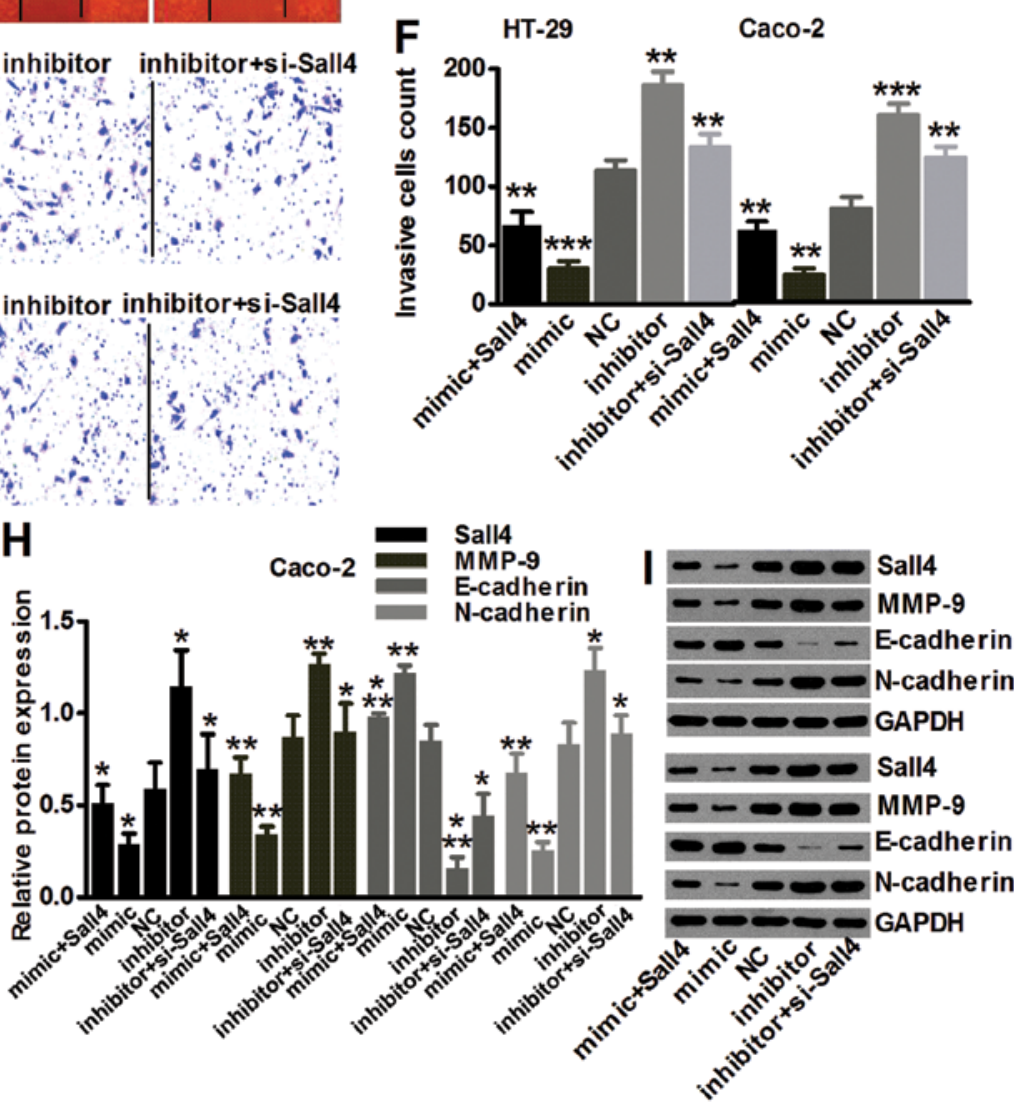

Figure 4. miR-219-5p inhibited colon cancer migration and invasion by targeting Sall4. (A and B) Wound healing assay images among the five groups in both cell lines; (C) cell migration ratio among five groups in both cell lines based on wound healing assay; (D and E) Transwell assay figures among five groups in both cell lines; (F) invasive cell count at x200 with 4 fields in total calculation among five groups in both cell lines; $(\mathrm{G}$ and $\mathrm{H})$ invasive-related protein expression among five groups in both cell lines; (I) representative western blot figures; mutual comparison relationships: mimic+Sall4 vs. mimic; mimic vs. NC; NC vs. inhibitor; inhibitor vs. inhibitor+si-Sall4. Column, means; Bar, SD. ${ }^{*} \mathrm{P}<0.05 ;{ }^{* *} \mathrm{P}<0.01 ;{ }^{* * *} \mathrm{P}<0.001$.

and cleaved caspase-9 are three common apoptosis-inducing proteins. By western blot assay, all three proteins were upregulated in miR-219-5p activation, according to the results of the apoptosis analysis $(\mathrm{P}<0.05$; Fig. $5 \mathrm{E}$ and $\mathrm{F})$. Thus, we proved that miR-219-5p induced colon cancer apoptosis by targeting Sall4.
miR-219-5p reduces colon cancer drug resistance by targeting Sall4. Drug resistance of colon cancer greatly restricted the clinical efficacy. We analyzed resistance of fluorouracil and oxaliplatin among five different groups in both cell lines, with these commonly used chemotherapy drugs in clinical practice. The results suggested that in both cell lines, miR-219-5p 

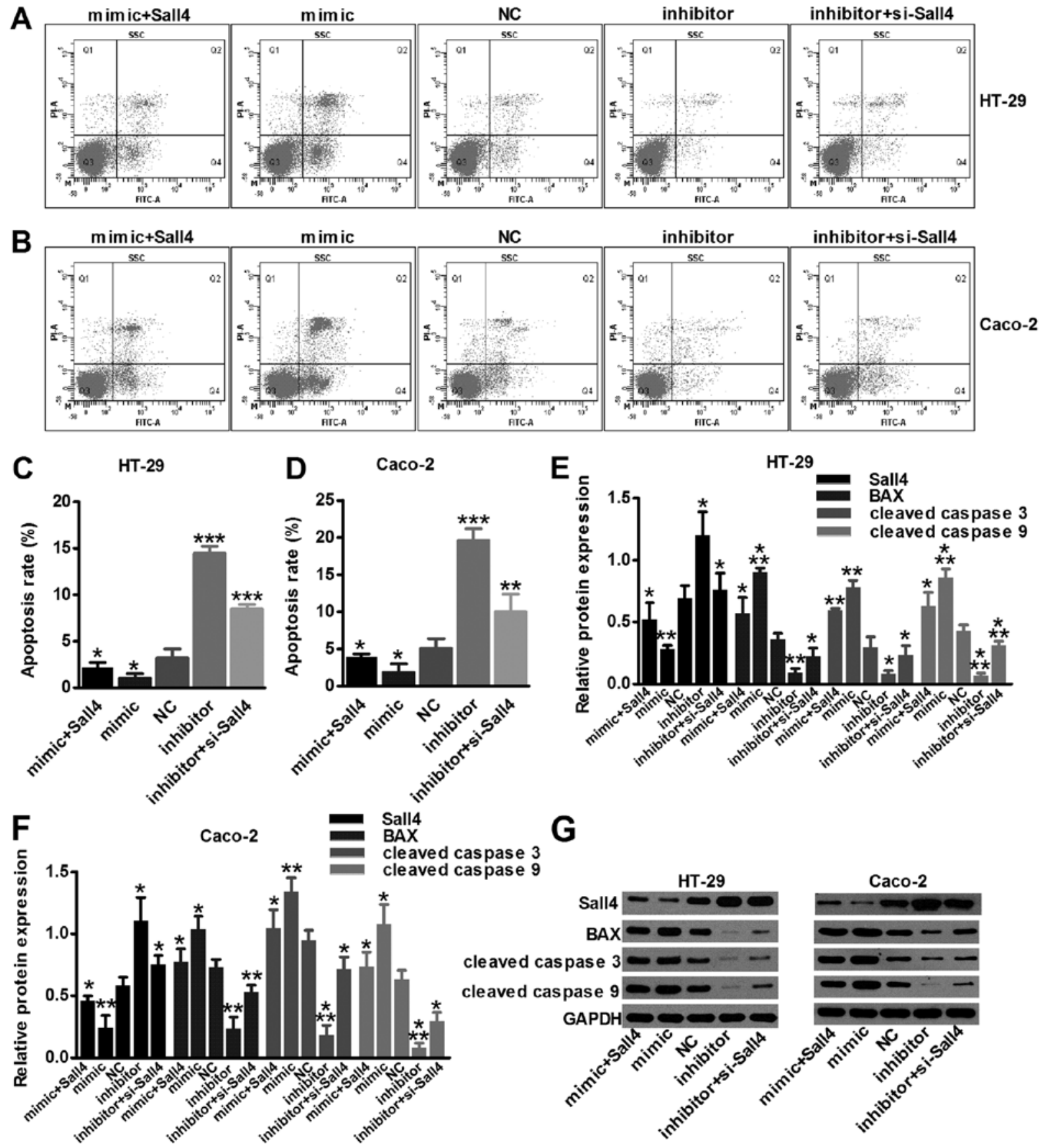

Figure 5. miR-219-5p induces colon cancer apoptosis by targeting Sall4. (A and B) Flow cytometry apoptosis among five groups in both cell lines; (C and D) apoptosis rate among the five groups in both cell lines based on wound healing assay; (E and F) apoptosis-related protein expression among five groups in both cell lines; (G) representative western blot figures; mutual comparison relationships: mimic+Sall4 vs. mimic; mimic vs. NC; NC vs. inhibitor; inhibitor vs. inhibitor+si-Sall4. Column, means; Bar, SD. ${ }^{*} \mathrm{P}<0.05 ;{ }^{* *} \mathrm{P}<0.01 ;{ }^{* * *} \mathrm{P}<0.001$

reduced colon cancer drug resistance by targeting Sall4 with greatly less viable cells after drug intervention $(\mathrm{P}<0.05$; Fig. 6A and B). P-gp and MRP1 are two representative proteins participating in chemotherapy resistance. Our western blot results confirmed the results of cell survival after drug intervention showing more expressed resistant proteins $(\mathrm{P}<0.05$; Fig. 6C and D).

\section{Discussion}

Although surgical operation functions as a preferred method in most colon cancer cases, those who suffer of locally advanced tumors or distant metastasis may be treated with internal medications, such as chemotherapy and specific targeted drugs. Targeted therapy is a future trend for advanced colon cancer medication as it is more specific and has less adverse effect compared to traditional chemotherapy drugs $(14,15)$. However, unlike the revolutionary role of imatinib on gastrointestinal stromal tumor (16) and trastuzumab on breast carcinoma treatment (17), curative targeted drugs for advanced colon cancer are still lacking. Classical chemotherapy drugs fluorouracil and oxaliplatin still dominate the adjunctive treatment (18), while recent clinical evidence have disclosed conclusions that certain indicative targeted drugs such as cetuximab $(19,20)$ 
A

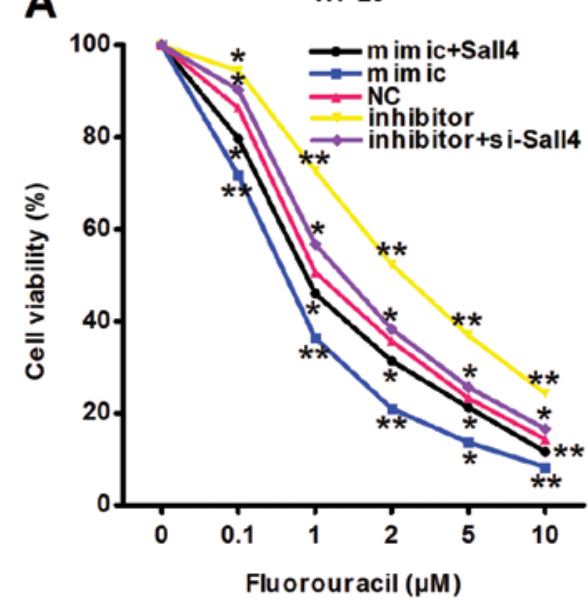

B

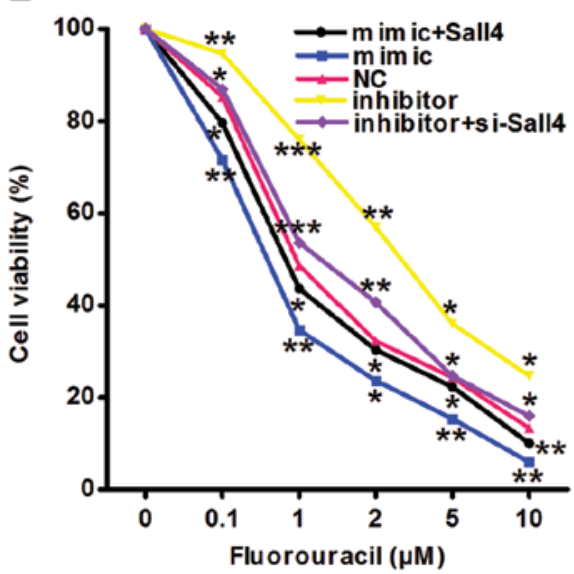

C

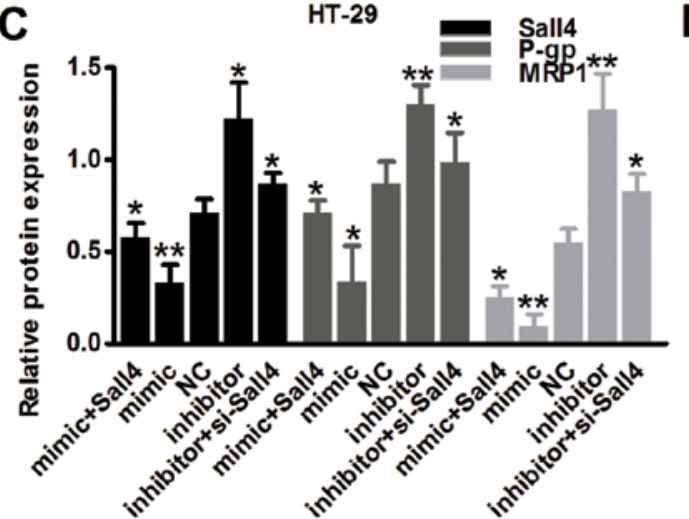

HT-29

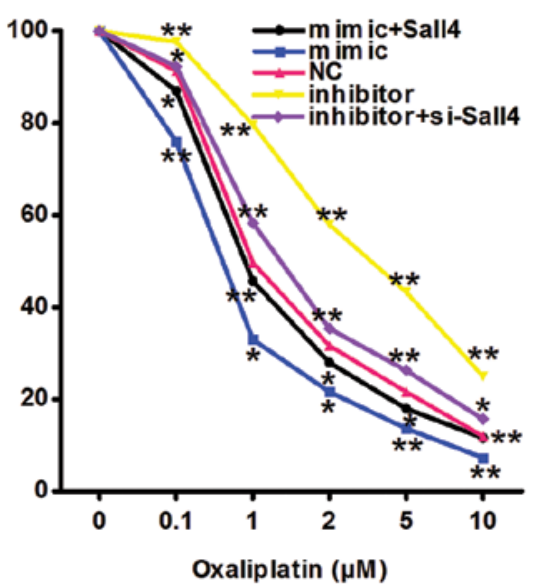

Caco-2

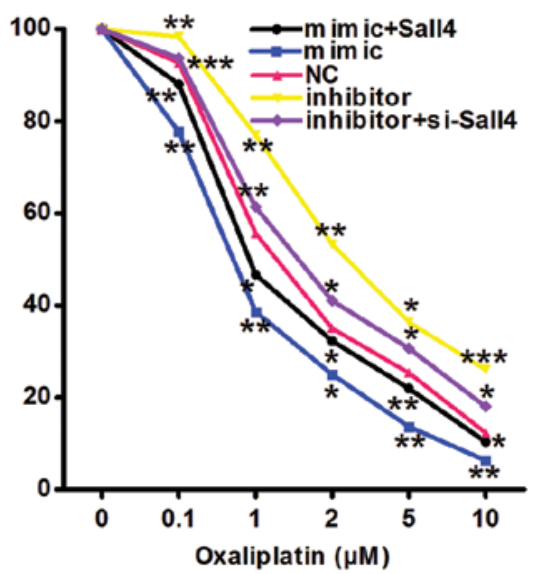

Caco-2
E

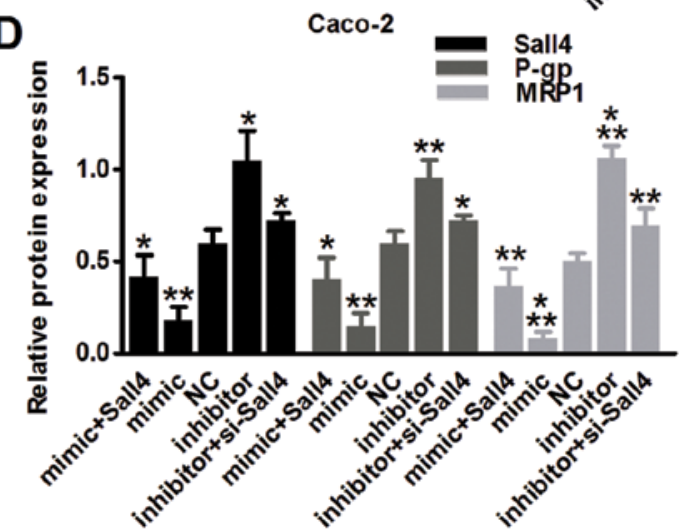

Figure 6. miR-219-5p reduced colon cancer drug resistance by targeting Sall4. (A and B) Cell survival rate diaphragms among five different groups in both cell lines based on CCK-8 test; (C and D) resistant-related protein expression among five different groups in both cell lines; (E) representative western blot figures; mutual comparison relationships: mimic+Sall4 vs. mimic; mimic vs. NC; NC vs. inhibitor; inhibitor vs. inhibitor+si-Sall4. Column, means; Bar, SD. ${ }^{*} \mathrm{P}<0.05 ;{ }^{* * *} \mathrm{P}<0.01 ;{ }^{* * * *} \mathrm{P}<0.001$.

and bevacizumab (21) demonstrate disappointing nonprofit survival rate in advanced patients despite the much expected clinical success on other malignancies such as lung cancer (22).

Search for available targets is a hotspot on colon cancer research, and miRNAs are a group of non-coding RNAs, acting as a broad regulation medium to connect cell signaling transduction and control cancer gene expression. Moreover, they can be readily transfected into target tissues by current techniques, facilitating its potential clinical usage. Those features have turn the miRNAs into a new favorite on targeted therapy research, which is proven by accumulating evidence $(3,4)$. The expression and function of miR-219-5p on colon cancer has not been published yet, and our experiments first revealed the antitumor properties of miR-219-5p on colon cancer via inhibiting the Boncogene Sall4 expression. This result is in coordination with the tumor suppressor role of miR-219-5p in liver cancer (7), papillary thyroid carcinoma (8) and glioblastoma (9) published already, suggesting its extensive anticancer efficacy. Many miRNAs have bipolar regulatory mechanisms 
in different types of cancers via targeting opposite functional genes, such as the oncogenic effect of miR-182 on colorectal cancer (23) while suppressing the proliferation of gastric cancer as well (24). Current evidence merely unveils the tumor inhibitory role of miR-219-5p, whether it still has the oncogenic role in diverse tumors or certain sub-types of colon cancer remains unclear. Additionally, miRNAs can be partially regulated by upstream effectors to precisely control its biological behavior, especially some classic developmental signaling pathways such as Sonic hedgehog (25) and Wnt/ $\beta$-catenin signaling (26). The network in which miR-219-5p is located and fits into all require further exploration before its clinical applications.

Sall4, a novel zinc finger transcriptional factor, behaves as an oncogene mediating tumorigenesis in different types of cancers, especially in liver cancer (27)and leukemia (28). The transcriptional role of Sall4 expands its downstream cancer gene expression to fulfill oncogenic efficacy, such as directly stimulating the expression of ubiquitous oncogene Bmi-1 in various cancers (29). Apart from the reliable evidence revealing its role in colon carcinogenesis and diagnosis $(10,11)$, Our experiments further verified that Sall4 overexpression correlated positively with chemo-resistance by upregulation of certain drug resistant genes including P-gp and MRP1, exhibiting a broader pro-oncogenic function of Sall4. Sall4 treatment has displayed inspiring tumor attenuating effect on leukemia cells, suggesting its potentials on clinical practice (30). Clarification of the mechanism network mediating its oncogenic role and regulating its expression is essential before launching the Sall4-targeted therapy. Several upstream signaling and miRNAs have been confirmed to participate in regulating expression of Sall4 including Wnt/ $\beta$-catenin signaling (12). miR-107 is reported to target Sall4 and to inhibit cell proliferation in glioma (13) and our experiment result verifies that miR-219-5p targets Sall4 in colon cancer to inhibit tumor progression and to reduce malignant properties. Furthermore, an oncogene can always be regulated by several different miRNAs such as YAP1 targeted by miR-15a (31), miR-375 (32) and miR200a (33). Thus, our laboratory will testi more potential miRNAs of Sall4 according to TargetScan prediction, offering more options of future miRNA mimic treatment against Sall4 on colon cancer.

The first miRNA targeted drug SPC3649 (anti-miR-122) was launched for clinical testing in 2008 and exhibits good clinical outcome against hepatitis $\mathrm{C}$ patients (34). Along with the breakthrough on pharmaceutical technology, certain miRNAs may appear on the market for clinical practice facilitating patients and clinicians. Thus, we need to make great efforts to elaborate the working systems of miR-219-5p and Sall4. The actual clinical efficacy of anti-miR-219-5p or anti-Sall4 therapy compared to current first-line medications needs further investigation under strict and comparable trials. We hope that it will bring benefits to patients in the future.

\section{Acknowledgements}

We sincerely appreciate what our team members have done for these experimente and the accomplishment is the fruit of great effort. We are also thankful for financial support by the
National Natural Science Foundation of China grant number 81172294.

\section{References}

1. Siegel RL, Miller KD and Jemal A: Cancer statistics, 2015. CA Cancer J Clin 65: 5-29, 2015.

2. Li L and Ma BB: Colorectal cancer in Chinese patients: Current and emerging treatment options. Onco Targets Ther 7: 1817-1828, 2014.

3. Tokarz P and Blasiak J: The role of microRNA in metastatic colorectal cancer and its significance in cancer prognosis and treatment. Acta Biochim Pol 59: 467-474, 2012.

4. Nana-Sinkam SP and Croce CM: Clinical applications for microRNAs in cancer. Clin Pharmacol Ther 93: 98-104, 2013.

5. Cui Y, Bai Y, Wang XD, Liu B, Zhao Z and Wang LS: Differential expression of miRNA in rat myocardial tissues under psychological and physical stress. Exp Ther Med 7: 901-906, 2014.

6. Shim SM, Jung SY, Nam HY, Kim HR, Lee MH, Kim JW, Han BG and Jeon JP: Network signatures of cellular immortalization in human lymphoblastoid cell lines. Biochem Biophys Res Commun 441: 438-446, 2013.

7. Huang N, Lin J, Ruan J, Su N, Qing R, Liu F, He B, Lv C, Zheng D and Luo R: MiR-219-5p inhibits hepatocellular carcinoma cell proliferation by targeting glypican-3. FEBS Lett 586: 884-891, 2012.

8. Huang C, Cai Z, Huang M, Mao C, Zhang Q, Lin Y, Zhang X, Tang B, Chen Y, Wang X, et al: miR-219-5p modulates cell growth of papillary thyroid carcinoma by targeting estrogen receptor $\alpha$. J Clin Endocrinol Metab 100: E204-E213, 2015.

9. Rao SA, Arimappamagan A, Pandey P, Santosh V, Hegde AS Chandramouli BA and Somasundaram K: miR-219-5p inhibits receptor tyrosine kinase pathway by targeting EGFR in glioblastoma. PLoS One 8: e63164, 2013.

10. Forghanifard MM, Moghbeli M, Raeisossadati R, Tavassoli A, Mallak AJ, Boroumand-Noughabi S and Abbaszadegan MR: Role of SALL4 in the progression and metastasis of colorectal cancer. J Biomed Sci 20: 6, 2013

11. Ardalan Khales S, Abbaszadegan MR, Abdollahi A, Raeisossadati R, Tousi MF and Forghanifard MM: SALL4 as a new biomarker for early colorectal cancers. J Cancer Res Clin Oncol 141: 229-235, 2015.

12. Böhm J, Sustmann C, Wilhelm C and Kohlhase J: SALL4 is directly activated by TCF/LEF in the canonical Wnt signaling pathway. Biochem Biophys Res Commun 348: 898-907, 2006.

13. He J, Zhang W, Zhou Q, Zhao T, Song Y, Chai L and Li Y: Low-expression of microRNA-107 inhibits cell apoptosis in glioma by upregulation of SALL4. Int J Biochem Cell Biol 45: 1962-1973, 2013.

14. Prenen H, Vecchione L and Van Cutsem E: Role of targeted agents in metastatic colorectal cancer. Target Oncol 8: 83-96, 2013.

15. Oestreicher P: Sequencing therapies: The role of targeted agents in metastatic colorectal cancer. ONS Connect 22 (Suppl 8): 37-38, 2007.

16. Iqbal $\mathrm{N}$ and Iqbal $\mathrm{N}$ : Imatinib: A breakthrough of targeted therapy in cancer. Chemother Res Pract 2014: 357027, 2014.

17. Balduzzi S, Mantarro S, Guarneri V, Tagliabue L, Pistotti V, Moja L and D'Amico R: Trastuzumab-containing regimens for metastatic breast cancer. Cochrane Database Syst Rev 6: CD006242, 2014.

18. Gustavsson B, Carlsson G, Machover D, Petrelli N, Roth A, Schmoll HJ, Tveit KM and Gibson F: A review of the evolution of systemic chemotherapy in the management of colorectal cancer. Clin Colorectal Cancer 14: 1-10, 2015.

19. Alberts SR, Sargent DJ, Nair S, Mahoney MR, Mooney M, Thibodeau SN, Smyrk TC, Sinicrope FA, Chan E, Gill S, et al: Effect of oxaliplatin, fluorouracil, and leucovorin with or without cetuximab on survival among patients with resected stage III colon cancer: A randomized trial. JAMA 307: 1383-1393, 2012.

20. Tveit KM, Guren T, Glimelius B, Pfeiffer P, Sorbye H, Pyrhonen S, Sigurdsson F, Kure E, Ikdahl T, Skovlund E, et al: Phase III trial of cetuximab with continuous or intermittent fluorouracil, leucovorin, and oxaliplatin (Nordic FLOX) versus FLOX alone in first-line treatment of metastatic colorectal cancer: The NORDIC-VII study. J Clin Oncol 30: 1755-1762, 2012.

21. Seymour MT: Adjuvant bevacizumab in colon cancer: Where did we go wrong? Lancet Oncol 13: 1176-1177, 2012.

22. Sculier JP, Berghmans T and Meert AP: Advances in target therapy in lung cancer. Eur Respir Rev 24: 23-29, 2015. 
23. Zhang $\mathrm{Y}$, Wang $\mathrm{X}$, Wang Z, Tang $\mathrm{H}$, Fan $\mathrm{H}$ and Guo Q: miR-182 promotes cell growth and invasion by targeting forkhead box F2 transcription factor in colorectal cancer. Oncol Rep 33: 2592-2598, 2015.

24. Tang L, Chen F, Pang EJ, Zhang ZQ, Jin BW and Dong WF: MicroRNA-182 inhibits proliferation through targeting oncogenic ANUBL1 in gastric cancer. Oncol Rep 33: 1707-1716, 2015.

25. Jiang Z, Cushing L, Ai X and Lü J: miR-326 is downstream of Sonic hedgehog signaling and regulates the expression of Gli2 and smoothened. Am J Respir Cell Mol Biol 51: 273-283, 2014.

26. Leung WK, He M,Chan AW, Law PT and Wong N: Wnt/3-Catenin activates MiR-183/96/182 expression in hepatocellular carcinoma that promotes cell invasion. Cancer Lett 362: 97-105, 2015.

27. Yong KJ, Gao C, Lim JS, Yan B, Yang H, Dimitrov T, Kawasaki A, Ong CW, Wong KF, Lee S, et al: Oncofetal gene SALL4 in aggressive hepatocellular carcinoma. N Engl J Med 368: 2266-2276, 2013.

28. Gao C, Kong NR and Chai L: The role of stem cell factor SALL4 in leukemogenesis. Crit Rev Oncog 16: 117-127, 2011.

29. Yang J, Chai L, Liu F, Fink LM, Lin P, Silberstein LE, Amin HM, Ward DC and Ma Y: Bmi-1 is a target gene for SALL4 in hematopoietic and leukemic cells. Proc Natl Acad Sci USA 104: 10494-10499, 2007.
30. Gao C, Dimitrov T, Yong KJ, Tatetsu H, Jeong HW, Luo HR, Bradner JE, Tenen DG and Chai L: Targeting transcription factor SALL4 in acute myeloid leukemia by interrupting its interaction with an epigenetic complex. Blood 121: 1413-1421, 2013.

31. Kang W, Tong JH, Lung RW, Dong Y, Zhao J, Liang Q, Zhang L, Pan Y, Yang W, Pang JC, et al: Targeting of YAP1 by microRNA15a and microRNA-16-1 exerts tumor suppressor function in gastric adenocarcinoma. Mol Cancer 14: 52, 2015.

32. Zhang ZW, Men T, Feng RC, Li YC, Zhou D and Teng CB: miR-375 inhibits proliferation of mouse pancreatic progenitor cells by targeting YAP1. Cell Physiol Biochem 32: 1808-1817, 2013.

33. Yu SJ, Hu JY, Kuang XY, Luo JM, Hou YF, Di GH, Wu J, Shen ZZ, Song HY and Shao ZM: MicroRNA-200a promotes anoikis resistance and metastasis by targeting YAP1 in human breast cancer. Clin Cancer Res 19: 1389-1399, 2013.

34. Gebert LF, Rebhan MA, Crivelli SE, Denzler R, Stoffel M and Hall J: Miravirsen (SPC3649) can inhibit the biogenesis of miR-122. Nucleic Acids Res 42: 609-621, 2014. 